\title{
MCP-1-induced protein-1, an immune regulator
}

\author{
Jiwei Xu", Sheng Fu", Wei Peng, Zihe Rao ${ }^{\star}$ \\ National Laboratory of Biomacromolecules, Institute of Biophysics, Chinese Academy of Sciences, Beijing 100101, China \\ $\square$ Correspondence: raozh@xtal.tsinghua.edu.cn \\ Received August 3, 2012 Accepted September 16, 2012
}

\begin{abstract}
MCP-1-induced protein-1 (MCPIP1) is a newly identified protein that is crucial to immune regulation. Mice lacking MCPIP1 gene suffer from severe immune disorders, and most of them cannot survive longer than 12 weeks. Considerable progress has been made in revealing the mechanism underlying the immune regulatory function of MCPIP1. MCPIP1 can act as an RNase to promote the mRNA degradation of some inflammatory cytokines, such as IL-6 and IL-1. Pre-microRNAs are also confirmed to be the substrate of MCPIP1 RNase. The structure of MCPIP1 N-terminal conserved domain shows a PilT N-terminus-like RNase structure, further supporting the notion that MCPIP1 has RNase activity. MCPIP1 can also deubiquitinate TNF receptor-associated factor family proteins, which are known to mediate immune and inflammatory responses. In this review, we summarize recent progress on the immune regulatory role of MCPIP1 and discuss the mechanisms underlying its function.
\end{abstract}

KEYWORDS MCPIP1, immune regulation, RNase, deubiquitinating enzyme, crystal structure

\section{INTRODUCTION}

MCP-1-induced protein-1 (MCPIP1), encoded by the gene $z c 3 h 12 a$, is a recently identified zinc-finger-containing protein that acts as a critical regulator of immune homeostasis. MCPIP1 is induced by MCP-1 in human peripheral blood monocytes or by IL-1 $\beta$ in human monocyte-derived macrophages through the activation of the NF-KB signaling pathway (Skalniak et al., 2009). MCPIP1 can also be induced by lipopolysaccharide (LPS) in mouse macrophages (Liang et al.,

\footnotetext{
*These authors contributed equally to the work.
}

2008; Matsushita et al., 2009).

The full-length protein of MCPIP1 has 599 amino acids; it contains an N-terminal conserved domain (NCD), a zinc-finger domain, and two proline-rich domains, as shown in Fig. 1. The NCD (residues 100-300) is conserved within the MCPIP family (MCPIP1, MCPIP2, MCPIP3, and MCPIP4) and among different species, indicating its functional importance. Sequence alignment (Fig. 1B) shows that the potential UBA (Liang et al., 2010), zinc-finger motif, and C-terminal are conserved within the MCPIP family, indicating the similarity of their functions. The NCD is suggested to be an RNase because it shares remote homology with the PilT N-terminal (PIN) domain (Matsushita et al., 2009). Further structural study by our group confirms that the NCD of MCPIP1 is a $\mathrm{PIN}$-like RNase. The zinc-finger motif in the MCPIP family is $\mathrm{CCCH}$ type with $\mathrm{CX}_{5} \mathrm{CX}_{5} \mathrm{CX}_{3} \mathrm{H}$ arrangement, which differs from the most commonly found $\mathrm{CX}_{8} \mathrm{CX}_{5} \mathrm{CX}_{3} \mathrm{H}$-type in other proteins. The $\mathrm{CCCH}$ zinc-finger motif is believed to interact with the AU-rich element located in the 3'-UTR of mRNA (Hake et al., 1998; Lai et al., 2002; Hudson et al., 2004). Two proline-rich domains exist in the MCPIP1 sequence. One lies in the N-terminal part and partially overlaps with the NCD (residues 100-126), and the other lies in the C-terminal of MCPIP1 (residues 458-536). These two proline-rich domains, which are not conserved in the MCPIP family, are thought to be responsible for its activation and protein-protein interactions (Zhou et al., 2006).

MCPIP1 participates in many different pathways and has multiple functions. The substrates of MCPIP1 and their functions are summarized in Table 1. First, it is identified as a transcription factor and proved to regulate the expression of multiple genes involved in different aspects of immune response (Zhou et al., 2006; Liang et al., 2008; Niu et al., 2008). MCPIP1 negatively regulates the LPS-induced promoter activity of TNFa and iNOS (Liang et al., 2008). It can also 
A

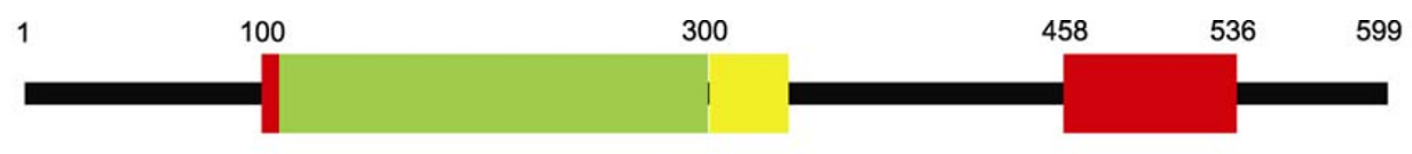

NCD

Zinc-finger motif

Proline-rich region

B

UBA

MCPIP1

MCPIP2

MCPIP3

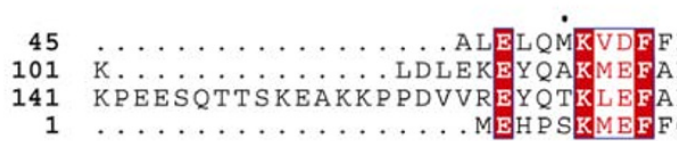

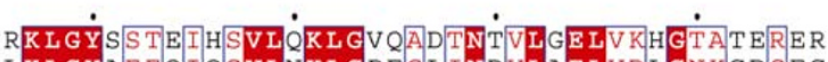
LKL G YAE E I Q SVLNKL GPES L INDVLAELVRLGNKGDSEG MCPIP4

NCD

MCPIPI MCPIP MCPIP3 MCPIP4

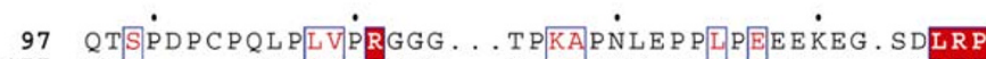

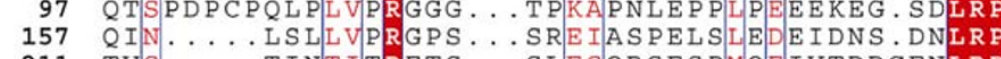

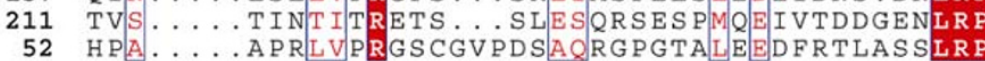
VIDGSNVAMSHGNKEVFSCRGİLL
VVIDGSNVAMSHGNKEEFSCRGI LL
IVIDGSNVAMSHGNKEVFSCRGIKL
IVIDGSNVAMSHGNKETFSCRGIKL

MCPIP1 163 MCPIP2 218 MCPIP3 273 MCPIP4 117
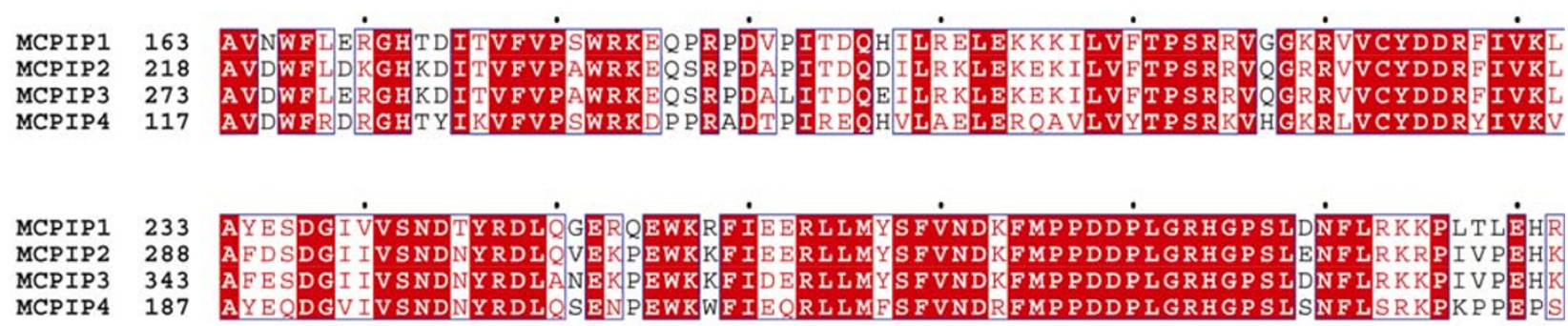

ZF

MCPIP1 303 MCPIP2 358 MCPIP3 413

MCPIP4 257

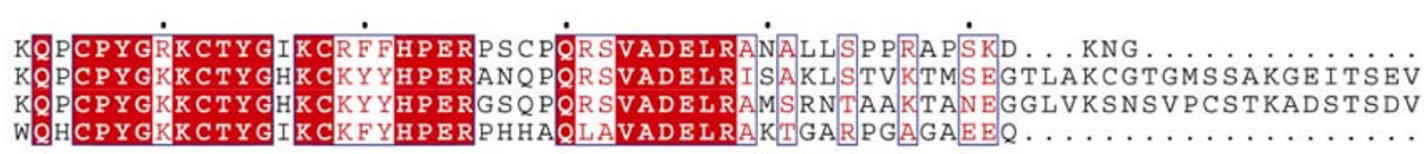
C-terminus MCPIP1 538 MCPIP2 771 MCPIP3 819 MCPIP4

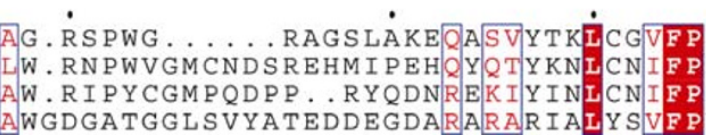

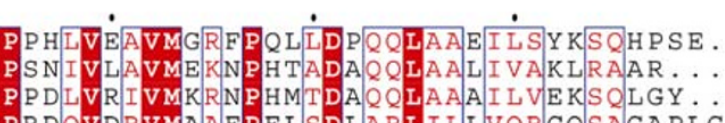

Figure 1. Sequence information of MPCIP1. (A) Schematic domain structure of MCPIP1. The NCD is green. The zinc-finger motif following the NCD is yellow. The two proline-rich regions located in the N-terminal and C-terminal are red. (B) Conserved sequences of MCPIP family proteins. MCPIP family proteins share a conserved UBA domain, NCD, ZF (zinc-finger motif), and C-terminal.

reportedly induce glial differentiation in NT2 cells by increasing GFAP expression and inducing astrocyte morphology (Vrotsos et al., 2009). Adipogenesis, a key differentiation process related to several diseases, can also be induced by MCPIP1 in 3T3-L1 cells (Younce et al., 2009). Another study demonstrates that MCPIP1 knockout mice shows severe anemia and immune disorder (Matsushita et al., 2009). This phenomenon has also been found in other $\mathrm{CCCH}$ zinc-finger-containing proteins such as tristetraprolin (TTP) and Roquin (Taylor et al., 1996; Vinuesa et al., 2005; Yu et al., 2007). Recently, numerous studies have focused on the RNase activity of MCPIP1 targeting on the mRNAs of IL-6, IL-1 $\beta$ (Matsushita et al., 2009; Mizgalska et al., 2009), and pre-microRNAs (Suzuki et al., 2011). Another study shows that MCPIP1 can act as a deubiquitinating enzyme (DUB) targeting TNF receptor-associated factor (TRAF) family proteins (Liang et al., 2010). 
MCPIP1 expression sensitizes cells to apoptosis under stress by inhibiting the formation of stress granules. This function also depends on the DUB activity of MCPIP1 (Qi et al., 2011). An overview of the functions of MPCIP1 is shown in Fig. 2.

Table 1 Substrates of MCPIP1 and their function in biological processes

\begin{tabular}{ll}
\hline Substrate & Function \\
\hline IL-6 mRNA & $\begin{array}{l}\text { Regulation of immune response, hematopoiesis, acute-phase response, and inflammation (Kishimoto, 1989, 2005; } \\
\text { Naugler and Karin, 2008). }\end{array}$ \\
IL-12p40 mRNA & Proinflammatory and immunostimulatory functions (Abdi, 2002; Khader et al., 2006). \\
IL-1ß mRNA & Inflammasome signaling and IFN-y or IL-10 production in TH17 cells (Rathinam et al., 2012; Zielinski et al., 2012). \\
pre-miR-135b & Up-regulation in colorectal cancer (Bandres et al., 2006). \\
pre-miR-146a & Down-regulation of TRAF6 and IRAK1 (O'Connell et al., 2012). \\
pre-miR-21 & Down-regulation of PDCD4; increased expression level in leukemia, lung cancer, breast cancer, etc. (Lee and Dutta, \\
& 2009; O'Connell et al., 2012). \\
pre-miR-155 & Down-regulation of SHIP1 and SOCS1; increased expression level in leukemia, pancreatic cancer, etc. (Lee and \\
pre-miR-143 & Dutta, 2009; O'Connell et al., 2012). \\
pre-miR-145 & Down-regulation of MACC1 and hexokinase 2 (Jiang et al., 2012; Zhang et al., 2012). \\
TRAF ubiquitination & Decreased expression level in colorectal and ovarian cancers (Lee and Dutta, 2009). \\
\hline
\end{tabular}

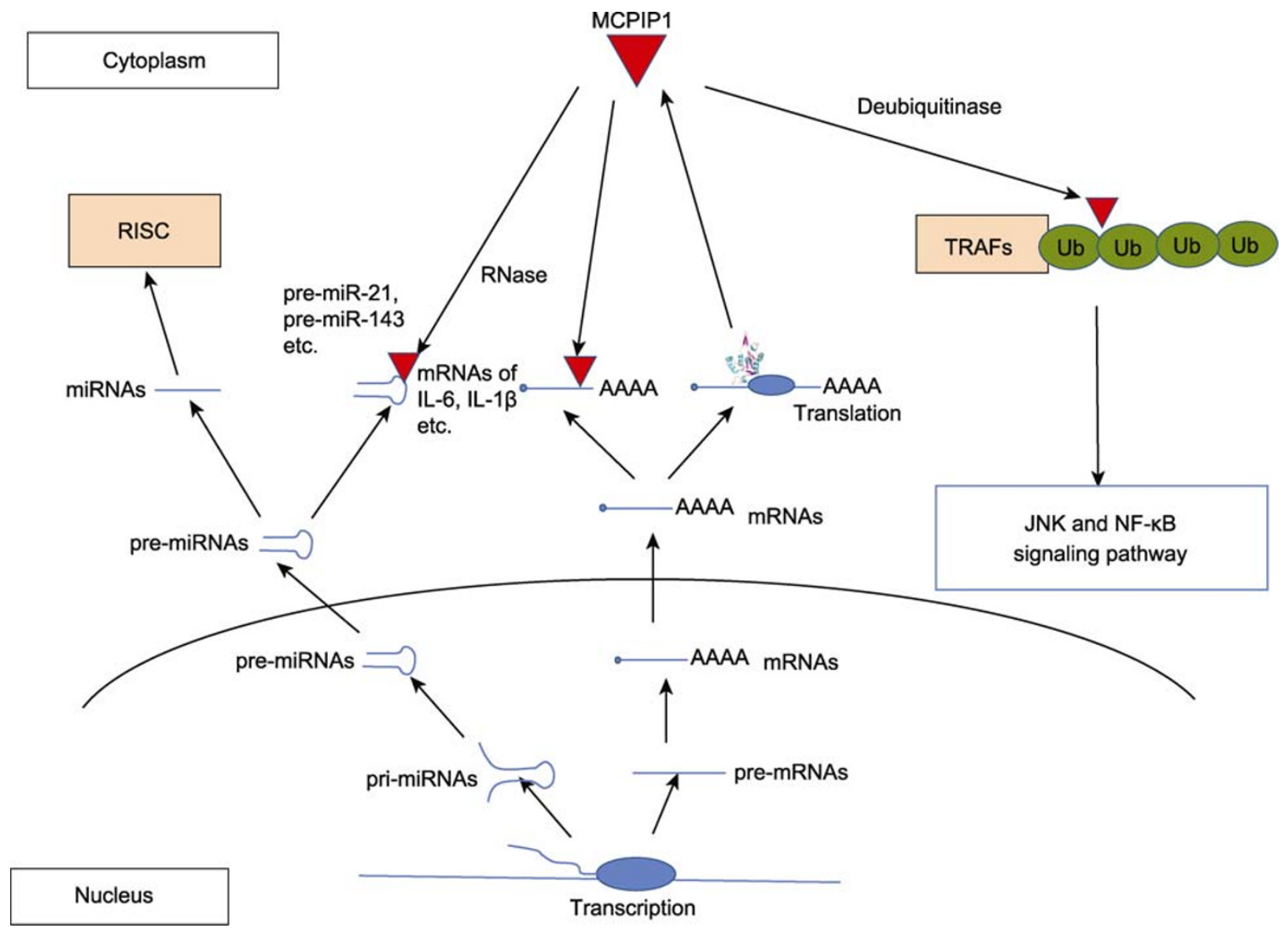

Figure 2. MCPIP1, an immune regulator. MCPIP1 can function as an RNase targeting pre-miRNAs and mRNAs of certain immune cytokines. MCPIP1 can also function as a DUB targeting TRAF family proteins, and thus down-regulates the JNK and NF-KB signaling pathways. 
The function of MCPIP1 as an immune regulator has been proven by many studies. This review discusses the immune regulatory function of MCPIP1, the progress made in its structural study, as well as the connection between the structure and function.

\section{TRANSCRIPTION FACTOR}

MCPIP1 has first been discovered as a transcription factor that possesses proapoptotic activity, and MCPIP1 overexpression leads to cell death in HEK293 cells or the cardiomyoblast cell line H9C2 (Zhou et al., 2006; Vrotsos et al., 2009). In MCPIP1-overexpressed HEK293 cells, some genes involved in cell death (such as Bar and TNFR2) are up-regulated, and MCPIP1 is found to be localized in the nucleus of these cells. MCPIP1 is also suggested to be involved in MCP-1-mediated angiogenesis by regulating the expression of several genes, such as cdh12 and cdh19 (Niu et al., 2008). However, Matsushita et al. (Matsushita et al., 2009) has later found that MCPIP1 is in the cytoplasm fraction rather than in the nuclear extract. Further investigation also reveals that MCPIP1 is localized in the cytoskeleton fraction, and some other proteins involved in mRNA metabolism have the same localization in cells (Henics, 1999; Mizgalska et al., 2009). More evidence is needed to prove the transcription factor activity of MCPIP1.

\section{RNASE ACTIVITY OF MCPIP1}

\section{IL-6 mRNA}

IL-6, produced primarily by $\mathrm{T}$ cells and macrophages, is a multifunction cytokine with a wide range of biological activities. IL-6 binding with its receptor initiates cellular events such as cell growth, gene activation, proliferation, survival, and differentiation (Kishimoto, 1989, 2005; Naugler and Karin, 2008). IL-6 is an important mediator in the acute-phase response. In response to a variety of signals such as bacterial pathogens, IL-6 plays an important role in the quick induction of an innate immune response, and thus contributes to host defense against pathogens. The dysregulation of IL-6 production leads to various autoimmune and chronic inflammatory diseases, including obesity, insulin resistance, autoimmune disorders, and inflammation-associated malignancies. IL-6-deficient mice also shows defective response to infections, impaired T-cell-regulated antibody response, and compromised inflammatory acute-phase response (Kopf et al., 1994). In clinical trials, the treatment of rheumatoid arthritis with humanized anti-IL-6 receptor antibody shows promising results (Tanaka et al., 2012). All these demonstrate the pathological significance of IL- 6 in various immune diseases.

MCPIP1 is proven to be an important regulator for the stability of IL-6 mRNA. The mRNA level of IL- 6 is increased in MCPIP1 knockout mice, suggesting a direct connection be- tween MCPIP1 and IL-6. MCPIP1 overexpression also shortens the half-life of IL-6 mRNA in macrophages (Matsushita et al., 2009). MCPIP1 is found to bind with and cleave the 3'-UTR of IL-6 mRNA. In contrast to TTP that recruits other nucleases to trigger mRNA destabilization, MCPIP1 alone is sufficient to induce IL-6 mRNA degradation (Anderson, 2008). A conserved element (about nucleotide 80-110) and five AU-rich elements exist in the 3'-UTR of IL-6 mRNA. Nucleotide $80-110$ is conserved among different species and can form a stem-loop structure (Paschoud et al., 2006). Sequence deletion in the 3'-UTR of IL-6 mRNA shows that the conserved element can affect the efficiency of degradation more than the AU-rich elements for the MCPIP1-mediated destabilization of IL-6 mRNA.

The NCD of MCPIP1 is responsible for IL-6 mRNA degradation. NCD shares remote homology with the PIN domain-like RNase. Several acidic residues, such as Asp141, are suggested to form a catalytic center according to the computational structure. The point mutation of Asp141 to Asn abolishes the RNase activity of MCPIP1. In our investigation, we have shown that the NCD itself is sufficient to digest RNA in vitro. We have also demonstrated that the zinc-finger motif and the first 112 residues in the $\mathrm{N}$-terminal domain are required for efficient RNA degradation by MCPIP1 (Xu et al., 2012).

MCPIP1-mediated IL-6 mRNA cleavage is an important immune regulatory mechanism that may protect an organism from immune disorders. Thus, MCPIP1 is a potential therapeutic target for the clinical treatment of immune diseases.

\section{mRNAs of other inflammatory cytokines}

Apart from IL-6, MCPIP1 also participates in the regulation of mRNAs of other inflammatory cytokines. The IL-1 $\beta$ mRNA level can be down-regulated by the overexpression of MCPIP1 or up-regulated by the siRNA inhibition of MCPIP1 (Mizgalska et al., 2009). The IL-12p40 level is also increased in MCPIP1 knockout macrophages (Matsushita et al., 2009), suggesting multiple mRNA targets of MCPIP1. The AU-rich element in the $3^{\prime}-$ UTR of IL-1 $\beta$ mRNA can affect its degradation activity. A putative stem-loop structure similar to the conserved element of IL-6 mRNA 3'-UTR may also account for the degradation of IL-1 $\beta$ RNA.

IL-1 $\beta$ induces MCPIP1 expression in human monocyte-derived macrophages. MCPIP1, in turn, degrades IL-1 $\beta$ mRNA, forming a negative feedback loop. Although different mRNA targets of MCPIP1 have been discovered, a common protein-RNA interaction pattern may exist during MCPIPmediated cytokine mRNA degradation.

\section{pre-microRNA}

MCPIP1 targets not only mRNAs but also microRNAs (miRNAs). miRNAs are small single-stranded RNAs ap- 
proximately 18-24 nucleotides long (http://microrna.sanger. ac.uk/). They play an important role in many biological processes, particularly in inflammatory pathways, primarily by the regulation of mRNA stability (Guo et al., 2010; O'Neill et al., 2011; O'Connell et al., 2012). Abnormal expression level of certain miRNAs is observed in cancers. Some miRNAs are identified as tumor suppressors or oncogenes in cancers (Garzon et al., 2009; Lee and Dutta, 2009).

miRNA biosynthesis begins with the transcription of pri-miRNA, which has a hairpin stem-loop structure. After transcription by RNA polymerase II, pri-miRNA is processed by a microprocessor to form pre-miRNA in the nucleus before being exported to the cytoplasm. The pre-miRNA is composed of a 22-base-pair stem, a loop, and a 3'-overhang (Lee et al., 2003; Denli et al., 2004; Gregory et al., 2004; Lee et al., 2004). This pre-miRNA is then processed by an RNase III enzyme, Dicer, to form a 22-nucleotide mature miRNA (Hutvagner et al., 2001). Mature miRNA subsequently forms a complex with RNA-induced silencing complex, and participates in the degradation of target mRNAs.

Recently, MCPIP1 is reported to down-regulate the expression level of several miRNAs (Suzuki et al., 2011). MCPIP1 can decrease the expression level of mature miRNA and pre-miRNA but shows no effect on pri-miRNA, indicating that MCPIP1 targets only pre-miRNAs. The direct targets of MCPIP1 include miR-135b, miR-146a, miR-21, miR-155, miR-143, and miR-145 (Suzuki et al., 2011). Most of them have important roles in biological processes as shown in Table 1. In cancer patients, miR-21 and miR-155, which both function as oncogenes, are found to be up-regulated (Volinia et al., 2006; Garzon et al., 2009). miR-155 promotes the immune response by down-regulating the expression levels of its targets, including SHIP1 and SOCS1, whereas miR- 146aand miR-21 attenuate the immune response (O'Connell et al., 2012). miR-143 targets hexokinase 2 to regulate cancer cell glycolysis, and also MACC1 to regulate cell invasion and migration (Jiang et al., 2012; Zhang et al., 2012). The regulation of these miRNA expression levels by MCPIP1 may be important for immune response, particularly for cancers.

The pre-miRNAs typically form a stem-loop structure. MCPIP1 reportedly targets the terminal loop region and cleaves miRNAs at the loop region as an endonuclease (Suzuki et al., 2011). Without the loop region, MCPIP1 fails to degrade miRNA.

In summary, a substantial amount of evidence proves the RNase activity of MCPIP1. However, the exact mechanism of MCPIP1 in miRNA level regulation requires further investigation.

\section{STRUCTURE OF MCPIP1 NCD AND ITS RNASE ACTIVITY}

Sequence analysis reveals that the NCD is an RNase. We have discovered that the NCD itself is sufficient for mRNA degradation in vitro. We have also determined the MCPIP1 NCD crystal structure at $2.0 \AA$ as shown in Fig. 3A (Xu et al., 2012). As previously suggested (Matsushita et al., 2009), MCPIP1 NCD is a PIN-like RNase that is supported by the superposition of the MCPIP1 NCD structure with the structure of a PIN domain family RNase, human SMG6 (pdb code: $2 \mathrm{hww}$ ). Although many differences exist among the overall structures, they share a similar catalytic center whose residues are highly conserved (Fig. 3B). The T5 5' exonuclease (pdb code: 1exn) is suggested to be similar with the MCPIP1 NCD structure by the DALI server (Holm and Rosenstrom, 2010). A similar $\alpha / \beta / \alpha$-sandwich fold of the overall structure

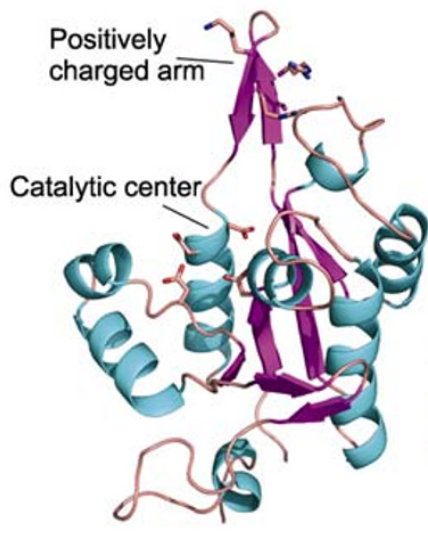

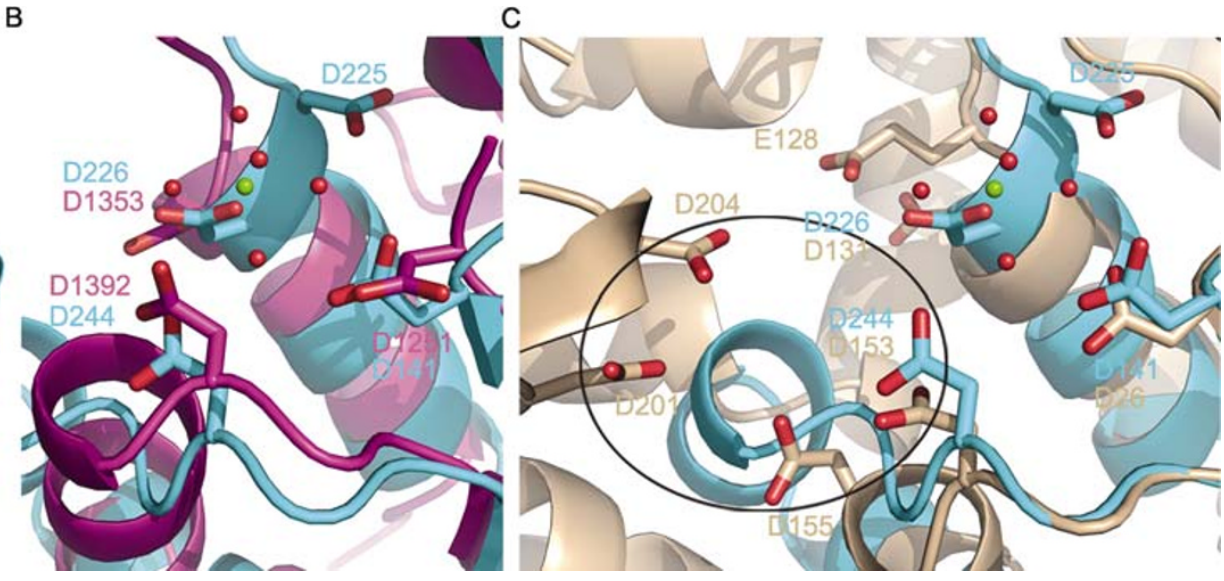

Figure 3. Structural study reveals the residues in MCPIP1 critical to its function. (A) Crystal structure of the MCPIP1 NCD. The catalytic center is composed of Asp141, Asp225, Asp226, and Asp244. A positively charged arm near the catalytic center, which is composed of Arg214, Arg215, Lys219, and Arg220, is presented. (B) Superposition of the catalytic centers of MCPIP1 and SMG6. The magnesium ion (green) in the structure of MCPIP1 NCD is surrounded by five water molecules (red) and Asp226. (C) Superposition of the catalytic centers of MCPIP1 and T5 5'-exonuclease. Residues D26, D128 D131, and D153 form a Mn ${ }^{2+}$ binding site, and residues D153, D155, D201, and D204 form another. No second metal-ion binding site (indicated by an oval) exists in the structure of the MCPIP1 NCD. 
and conserved catalytic center is also found in the superposition of the NCD structure with a T5 5' exonuclease structure (Fig. 3C). The conserved catalytic center in MCPIP1 is composed of four Asp residues: Asp141, Asp225, Asp226, and Asp244.

In the solved structure, we have shown a magnesium ion in the catalytic center surrounded by Asp226 and other five water molecules (Fig. 3B). The structure of the catalytic center reveals several residues that are crucial to stabilizing the magnesium ion and the surrounding water molecules. The importance of these residues is verified by an in vivo luciferase assay. Asp141, which is suggested to be crucial to RNase activity, is found in the catalytic center to be stabilizing the water molecules around the magnesium ion. We have also found a positively charged arm consisting Arg214, Arg215, Lys219, and Arg220 near the catalytic center, and confirmed the importance of these residues by site mutation.

Interestingly, the PIN domain RNase and the FEN-like nuclease are categorized into the two-metal ion nuclease family (Yang, 2011), whereas only one magnesium ion is found in the catalytic center of MCPIP1. Superposition of the MCPIP1 NCD structure with T5 $5^{\prime}$ exonuclease structure presents a good fit in one of the metal-ion binding site. However, in the structure of MCPIP1 NCD, no residue composition is similar to the second metal-ion binding site of $\mathrm{T} 55^{\prime}$ exonuclease (Fig. 3C).

\section{Substrate specificity}

Substrate specificity is needed for MCPIP1 to obtain the accuracy of immune regulation. Several studies suggest that the $\mathrm{CCCH}$-type zinc-finger motif can interact with the AU-rich elements located in the 3'-UTR of mRNAs (Lai et al., 2002; Hudson et al., 2004). This interaction is needed for the efficient MCPIP1 degradation of IL-6 mRNA, but is not a necessary condition. The AU-rich elements are common in the 3'-UTR of many different mRNAs. Apart from the interaction between the zinc-finger motif and AU-rich elements, other mechanisms may be required for substrate specificity.

IL-6 mRNA digestion by MCPIP1 needs the recognition of a conserved element in the $3^{\prime}$-UTR (about nucleotide 80-110). The conserved element in the 3'-UTR of IL-6 mRNA and several pre-miRNAs can form hairpin structures as predicted, and MCPIP1 cleaves the pre-miRNA at the unpaired region (Suzuki et al., 2011). The recognition of a secondary structure of mRNA may be important for substrate binding and its specificity. The positively charged arm near the catalytic center may play an important role in binding the hairpin structure of mRNA.

Other members of the MCPIP family (MCPIP2, MCPIP3, and MCPIP4) possess an NCD and a zinc-finger motif similar to MCPIP1, but they cannot cleave pre-miRNA. Oligomerization with MCPIP1 or interaction with other proteins may be needed for their substrate specificity.

\section{MCPIP1 DEUBIQUITINATES TRAF PROTEINS}

Another important regulatory function of MCPIP1 is its DUB activity. Ubiquitination is well known to be important for protein modification in the signal transduction of immune regulation (Liu et al., 2005; Bhoj and Chen, 2009). The K63-linked ubiquitination of the TRAF family proteins plays a significant role in the NF-KB signaling pathway, which is important for both innate and adaptive immune responses (Sun, 2008). MCPIP1 protein can act as DUB both in vivo and in vitro. MCPIP1 overexpression in HEK293 cells results in fewer ubiquitinated proteins than normal cells. Purified MCPIP1 protein can cleave both K48- and K63-linked polyubiquitin chains, and this DUB activity can be abolished by $N$-ethylmaleimide (Liang et al., 2010). With its deubiquitinase activity, MCPIP1 suppresses the LPS-mediated JNK and NF-KB signaling pathways. TRAF family proteins are proved to be the direct targets of MCPIP1 deubiquitinase (Liang et al., 2010). Functioning as a DUB, MCPIP1 also completely blocks LPS- and IL-1 $\beta$-induced JNK phosphorylation.

Until now, about 100 DUBs are found in the human genome. DUBs are proteases that can be categorized into five families according to their domain structures. Four families are Cys proteases, including ubiquitin C-terminal hydrolases, ovarian tumor proteases, ubiquitin-specific proteases, and Josephins. The other family is JAB1/MPN/MOV34 zinc metalloenzymes (Komander et al., 2009; Reyes-Turcu et al., 2009). The domain architecture and crystal structure of each family have been reviewed (Komander et al., 2009). However, we have not found evidence that MCPIP1 belongs to any of these families. The crystal structure of MCPIP1 NCD shares no similarity with any known DUBs structure. The N-terminal of MCPIP1, which is important for its deubiquitinase activity as suggested by a previous study (Liang et al., 2010), may be a novel DUB domain different from the known five families of DUBs. The full-length structure of MCPIP1 can provide more information on DUB activity. A sequence study suggests a potential UBA domain located in the N-terminal of MCPIP1 (residues 40-90), and this domain is conserved in the MCPIP family (Fig. 1B).

\section{CONCLUSION}

MCPIP1, functioning as an RNase and DUB, is an important immune regulator (Fig. 2). Numerous studies show that MCPIP1 is involved in many processes, such as the promotion of angiogenesis (Niu et al., 2008), inhibition of macrophage activation (Liang et al., 2008), attenuation of myocardial dysfunction (Niu et al., 2011), and induction of cardiomyoblast death (Younce and Kolattukudy, 2010). The RNase and deubiquitinase activities of MCPIP1 are well studied, and the NCD structure is solved. In this review, we conclude that MCPIP1 plays an important role in immune response regulation by directly targeting the mRNAs of several inflammatory 
cytokines, some pre-miRNAs, and the TRAF family proteins. Significant progress has been achieved in the functional and structural studies of MCPIP1 over the past few years. Given the promising of results of targeting the MCP-1/CCR2 pathway for cardiovascular disease therapy (Kolattukudy and Niu, 2012), targeting MCPIP1 may be a feasible method of treating chronic inflammatory diseases or cancers.

MCPIP1 plays an important role in immune regulation; thus, solving the full-length structure of MCPIP1 and conducting more functional studies can enable the complete characterization of MCPIP1. The full-length structure may help elucidate the substrate-binding mechanism and the function of the zinc-finger motif. According to the conserved sequences of the MCPIP family proteins as well as the similar function of MCPIP1 and MCPIP4, revealing the function of all MCPIP family proteins is important. The study on immune activity regulation of MCPIP1 needs further investigation.

\section{ACKNOWLEDGEMENTS}

This work was supported by grants from the National Basic Research Program (973 Program) (Nos. 2011CB915501 and 2011CB910304).

\section{ABBREVIATIONS}

DUB, deubiquitinating enzyme; LPS, lipopolysaccharide; MCPIP1, MCP-1-induced protein-1; NCD, N-terminal conserved domain; PIN, PilT N-terminal; TRAF, TNF receptor-associated factor

\section{REFERENCES}

Abdi, K. (2002). IL-12: the role of p40 versus p75. Scand J Immunol 56, 1-11.

Anderson, P. (2008). Post-transcriptional control of cytokine production. Nat Immunol 9, 353-359.

Bandres, E., Cubedo, E., Agirre, X., Malumbres, R., Zarate, R., Ramirez, N., Abajo, A., Navarro, A., Moreno, I., Monzo, M., et al. (2006). Identification by Real-time PCR of 13 mature microRNAs differentially expressed in colorectal cancer and non-tumoral tissues. Mol Cancer 5, 29.

Bhoj, V.G., and Chen, Z.J. (2009). Ubiquitylation in innate and adaptive immunity. Nature 458, 430-437.

Denli, A.M., Tops, B.B., Plasterk, R.H., Ketting, R.F., and Hannon, G.J. (2004). Processing of primary microRNAs by the Microprocessor complex. Nature 432, 231-235.

Garzon, R., Calin, G.A., and Croce, C.M. (2009). MicroRNAs in Cancer. Annu Rev Med 60, 167-179.

Gatot, J.S., Gioia, R., Chau, T.L., Patrascu, F., Warnier, M., Close, P., Chapelle, J.P., Muraille, E., Brown, K., Siebenlist, U., et al. (2007). Lipopolysaccharide-mediated interferon regulatory factor activation involves TBK1-IKKepsilon-dependent Lys(63)-linked polyubiquitination and phosphorylation of TANK/I-TRAF. J Biol Chem 282, 31131-31146.

Gregory, R.I., Yan, K.P., Amuthan, G., Chendrimada, T., Doratotaj, B., Cooch, N., and Shiekhattar, R. (2004). The Microprocessor complex mediates the genesis of microRNAs. Nature 432, 235-240.
Guo, H., Ingolia, N.T., Weissman, J.S., and Bartel, D.P. (2010). Mammalian microRNAs predominantly act to decrease target mRNA levels. Nature 466, 835-840.

Hake, L.E., Mendez, R., and Richter, J.D. (1998). Specificity of RNA binding by CPEB: requirement for RNA recognition motifs and a novel zinc finger. Mol Cell Biol 18, 685-693.

Henics, T. (1999). Microfilament-dependent modulation of cytoplasmic protein binding to TNFalpha mRNA AU-rich instability element in human lymphoid cells. Cell Biol Int 23, 561-570.

Holm, L., and Rosenstrom, P. (2010). Dali server: conservation mapping in 3D. Nucleic Acids Res 38, W545-549.

Hudson, B.P., Martinez-Yamout, M.A., Dyson, H.J., and Wright, P.E. (2004). Recognition of the mRNA AU-rich element by the zinc finger domain of TIS11d. Nat Struct Mol Biol 11, 257-264.

Hutvagner, G., McLachlan, J., Pasquinelli, A.E., Balint, E., Tuschl, T., and Zamore, P.D. (2001). A cellular function for the RNA-interference enzyme Dicer in the maturation of the let-7 small temporal RNA. Science 293, 834-838.

Jiang, S., Zhang, L.F., Zhang, H.W., Hu, S., Lu, M.H., Liang, S., Li, B., Li, Y., Li, D., Wang, E.D., et al. (2012). A novel miR-155/miR-143 cascade controls glycolysis by regulating hexokinase 2 in breast cancer cells. EMBO J 31, 1985-1998.

Khader, S.A., Partida-Sanchez, S., Bell, G., Jelley-Gibbs, D.M., Swain, S., Pearl, J.E., Ghilardi, N., Desauvage, F.J., Lund, F.E., and Cooper, A.M. (2006). Interleukin 12p40 is required for dendritic cell migration and T cell priming after Mycobacterium tuberculosis infection. J Exp Med 203, 1805-1815.

Kishimoto, T. (1989). The biology of interleukin-6. Blood 74, 1-10.

Kishimoto, T. (2005). Interleukin-6: from basic science to medicine--40 years in immunology. Annu Rev Immunol 23, 1-21.

Kolattukudy, P.E., and Niu, J. (2012). Inflammation, endoplasmic reticulum stress, autophagy, and the monocyte chemoattractant protein-1/CCR2 pathway. Circ Res 110, 174-189.

Komander, D., Clague, M.J., and Urbe, S. (2009). Breaking the chains: structure and function of the deubiquitinases. Nat Rev Mol Cell Biol 10, 550-563.

Kopf, M., Baumann, H., Freer, G., Freudenberg, M., Lamers, M., Kishimoto, T., Zinkernagel, R., Bluethmann, H., and Kohler, G. (1994). Impaired immune and acute-phase responses in interleukin-6-deficient mice. Nature 368, 339-342.

Lai, W.S., Kennington, E.A., and Blackshear, P.J. (2002). Interactions of $\mathrm{CCCH}$ zinc finger proteins with mRNA: non-binding tristetraprolin mutants exert an inhibitory effect on degradation of AU-rich element-containing mRNAs. J Biol Chem 277, 9606-9613.

Lee, Y., Ahn, C., Han, J., Choi, H., Kim, J., Yim, J., Lee, J., Provost, P., Radmark, O., Kim, S., et al. (2003). The nuclear RNase III Drosha initiates microRNA processing. Nature 425, 415-419.

Lee, Y., Kim, M., Han, J., Yeom, K.H., Lee, S., Baek, S.H., and Kim, V.N. (2004). MicroRNA genes are transcribed by RNA polymerase II. EMBO J 23, 4051-4060.

Lee, Y.S., and Dutta, A. (2009). MicroRNAs in cancer. Annu Rev Pathol 4, 199-227.

Liang, J., Saad, Y., Lei, T., Wang, J., Qi, D., Yang, Q., Kolattukudy, P.E., and Fu, M. (2010). MCP-induced protein 1 deubiquitinates TRAF proteins and negatively regulates JNK and NF-kappaB signaling. J Exp Med 207, 2959-2973.

Liang, J., Wang, J., Azfer, A., Song, W., Tromp, G., Kolattukudy, P.E., 
and $\mathrm{Fu}, \mathrm{M}$. (2008). A novel $\mathrm{CCCH}$-zinc finger protein family regulates proinflammatory activation of macrophages. J Biol Chem 283, 6337-6346.

Liu, Y.C., Penninger, J., and Karin, M. (2005). Immunity by ubiquitylation: a reversible process of modification. Nat Rev Immunol 5, 941-952.

Matsushita, K., Takeuchi, O., Standley, D.M., Kumagai, Y., Kawagoe, T., Miyake, T., Satoh, T., Kato, H., Tsujimura, T., Nakamura, H., et al. (2009). Zc3h12a is an RNase essential for controlling immune responses by regulating mRNA decay. Nature 458, 1185-1190.

Mizgalska, D., Wegrzyn, P., Murzyn, K., Kasza, A., Koj, A., Jura, J., and Jarzab, B. (2009). Interleukin-1-inducible MCPIP protein has structural and functional properties of RNase and participates in degradation of IL-1beta mRNA. FEBS J 276, 7386-7399.

Naugler, W.E., and Karin, M. (2008). The wolf in sheep's clothing: the role of interleukin- 6 in immunity, inflammation and cancer. Trends Mol Med 14, 109-119.

Niu, J., Azfer, A., Zhelyabovska, O., Fatma, S., and Kolattukudy, P.E. (2008). Monocyte chemotactic protein (MCP)-1 promotes angiogenesis via a novel transcription factor, MCP-1-induced protein (MCPIP). J Biol Chem 283, 14542-14551.

Niu, J., Wang, K., Graham, S., Azfer, A., and Kolattukudy, P.E. (2011). MCP-1-induced protein attenuates endotoxin-induced myocardial dysfunction by suppressing cardiac NF-small ka, CyrillicB activation via inhibition of Ismall ka, CyrillicB kinase activation. J Mol Cell Cardiol 51, 177-186.

O'Connell, R.M., Rao, D.S., and Baltimore, D. (2012). microRNA regulation of inflammatory responses. Annu Rev Immunol 30, 295-312.

O'Neill, L.A., Sheedy, F.J., and McCoy, C.E. (2011). MicroRNAs: the fine-tuners of Toll-like receptor signalling. Nat Rev Immunol 11, 163-175.

Paschoud, S., Dogar, A.M., Kuntz, C., Grisoni-Neupert, B., Richman, L., and Kuhn, L.C. (2006). Destabilization of interleukin-6 mRNA requires a putative RNA stem-loop structure, an AU-rich element, and the RNA-binding protein AUF1. Molecular and cellular biology $26,8228$.

Qi, D., Huang, S., Miao, R., She, Z.G., Quinn, T., Chang, Y., Liu, J., Fan, D., Chen, Y.E., and Fu, M. (2011). Monocyte chemotactic protein-induced protein 1 (MCPIP1) suppresses stress granule formation and determines apoptosis under stress. J Biol Chem 286, 41692-41700.

Rathinam, V.A., Vanaja, S.K., and Fitzgerald, K.A. (2012). Regulation of inflammasome signaling. Nat Immunol 13, 333-332.

Reyes-Turcu, F.E., Ventii, K.H., and Wilkinson, K.D. (2009). Regulation and cellular roles of ubiquitin-specific deubiquitinating enzymes. Annu Rev Biochem 78, 363-397.

Skalniak, L., Mizgalska, D., Zarebski, A., Wyrzykowska, P., Koj, A., and Jura, J. (2009). Regulatory feedback loop between NF-kappaB and MCP-1-induced protein 1 RNase. FEBS J 276, 5892-5905.

Sun, S.C. (2008). Deubiquitylation and regulation of the immune response. Nat Rev Immunol 8, 501-511.

Suzuki, H.I., Arase, M., Matsuyama, H., Choi, Y.L., Ueno, T., Mano,
H., Sugimoto, K., and Miyazono, K. (2011). MCPIP1 ribonuclease antagonizes dicer and terminates microRNA biogenesis through precursor microRNA degradation. Mol Cell 44, 424-436.

Tanaka, T., Narazaki, M., and Kishimoto, T. (2012). Therapeutic targeting of the interleukin-6 receptor. Annu Rev Pharmacol Toxicol 52, 199-219.

Taylor, G.A., Carballo, E., Lee, D.M., Lai, W.S., Thompson, M.J., Patel, D.D., Schenkman, D.I., Gilkeson, G.S., Broxmeyer, H.E., Haynes, B.F., et al. (1996). A pathogenetic role for TNF alpha in the syndrome of cachexia, arthritis, and autoimmunity resulting from tristetraprolin (TTP) deficiency. Immunity 4, 445-454.

Vinuesa, C.G., Cook, M.C., Angelucci, C., Athanasopoulos, V., Rui, L., Hill, K.M., Yu, D., Domaschenz, H., Whittle, B., Lambe, T., et al. (2005). A RING-type ubiquitin ligase family member required to repress follicular helper $T$ cells and autoimmunity. Nature 435, 452-458.

Volinia, S., Calin, G.A., Liu, C.G., Ambs, S., Cimmino, A., Petrocca, F., Visone, R., lorio, M., Roldo, C., Ferracin, M., et al. (2006). A microRNA expression signature of human solid tumors defines cancer gene targets. Proc Natl Acad Sci U S A 103, 2257-2261.

Vrotsos, E.G., Kolattukudy, P.E., and Sugaya, K. (2009). MCP-1 involvement in glial differentiation of neuroprogenitor cells through APP signaling. Brain Res Bull 79, 97-103.

Xu, J., Peng, W., Sun, Y., Wang, X., Xu, Y., Li, X., Gao, G., and Rao, Z. (2012). Structural study of MCPIP1 N-terminal conserved domain reveals a PIN-like RNase. Nucleic Acids Res. (In Press).

Yang, W. (2011). Nucleases: diversity of structure, function and mechanism. Rev Biophys 44, 1-93.

Younce, C.W., Azfer, A., and Kolattukudy, P.E. (2009). MCP-1 (monocyte chemotactic protein-1)-induced protein, a recently identified zinc finger protein, induces adipogenesis in 3T3-L1 pre-adipocytes without peroxisome proliferator-activated receptor gamma. J Biol Chem 284, 27620-27628.

Younce, C.W., and Kolattukudy, P.E. (2010). MCP-1 causes cardiomyoblast death via autophagy resulting from ER stress caused by oxidative stress generated by inducing a novel zinc-finger protein, MCPIP. Biochem J 426, 43-53.

Yu, D., Tan, A.H., Hu, X., Athanasopoulos, V., Simpson, N., Silva, D.G., Hutloff, A., Giles, K.M., Leedman, P.J., Lam, K.P., et al. (2007). Roquin represses autoimmunity by limiting inducible T-cell co-stimulator messenger RNA. Nature 450, 299-303.

Zhang, Y., Wang, Z., Chen, M., Peng, L., Wang, X., Ma, Q., Ma, F., and Jiang, B. (2012). MicroRNA-143 targets MACC1 to inhibit cell invasion and migration in colorectal cancer. Mol Cancer 11, 23.

Zhou, L., Azfer, A., Niu, J., Graham, S., Choudhury, M., Adamski, F.M., Younce, C., Binkley, P.F., and Kolattukudy, P.E. (2006). Monocyte chemoattractant protein-1 induces a novel transcription factor that causes cardiac myocyte apoptosis and ventricular dysfunction. Circ Res 98, 1177-1185.

Zielinski, C.E., Mele, F., Aschenbrenner, D., Jarrossay, D., Ronchi, F., Gattorno, M., Monticelli, S., Lanzavecchia, A., and Sallusto, F. (2012). Pathogen-induced human TH17 cells produce IFN-gamma or IL-10 and are regulated by IL-1beta. Nature 484, 514-518. 